\title{
$\alpha$-synuclein promotes progression of Parkinson's disease by upregulating autophagy signaling pathway to activate NLRP3 inflammasome
}

\author{
XIAOHONG WANG ${ }^{1}$, JINGHONG CHI ${ }^{1}$, DI HUANG ${ }^{2}$, LI DING ${ }^{1}$, \\ $\mathrm{XIAOJING} \mathrm{ZHAO}^{1}$, LAI JIANG ${ }^{1}$, YANG $\mathrm{YU}^{1}$ and FENG GAO ${ }^{1}$ \\ ${ }^{1}$ The 5th Department of Neurology, the Third Affiliated Hospital of Qiqihar Medical University; \\ ${ }^{2}$ The 8th Department of Neurology, the First Hospital of Qiqihar City, Qiqihar, Heilongjiang 161000, P.R. China
}

Received September 23, 2019; Accepted November 20, 2019

DOI: $10.3892 /$ etm.2019.8297

\begin{abstract}
Mechanism by which $\alpha$-synuclein affects the progression of Parkinson's disease through Pyrin Domain Containing Protein 3 (NLRP3) was explored. Peripheral blood plasma of 40 Parkinson's disease patients and 40 normal healthy people attending the department of neurology of the Third Affiliated Hospital of Qiqihar Medical University were collected from March 2018 to January 2019. The expression levels of oligomers, phosphorylated $\alpha$-synuclein, interleukin-1 $\beta$ (IL-1 $\beta$ ), interleukin-6 (IL-6) and transforming growth factor- $\alpha$ (TGF- $\alpha$ ) in plasma were detected by ELISA. Astrocytes in mouse brain tissues were extracted by primary culture method, the cells were divided into drug group and the drug + inhibitor group. After adding 0,5, 10 and $20 \mu \mathrm{g}$ oligomerized $\alpha$-synuclein or $5 \mathrm{mM}$ autophagy inhibitor 3-Methyladenine (3-MA), the expression level of NLRP3, caspase-1, IL-1 $\beta$ and Atg5 proteins in the cells was detected. The expression level of IL-1 $\beta$ in peripheral blood of PD patients was significantly increased $(0.604 \pm 0.136 \mu \mathrm{mol} / 1$ vs. $1.876 \pm 0.327 \mu \mathrm{mol} / 1, \mathrm{P}=0.002$ ), while there was no significant difference between IL- 6 and TGF- $\alpha$. Both oligomers $(0.171 \pm 0.045 \mu \mathrm{mol} / 1$ vs. $0.676 \pm 0.084 \mu \mathrm{mol} / 1, \mathrm{P}<0.0001)$ and phosphorylated $\alpha$-synuclein $(0.128 \pm 0.041 \mu \mathrm{mol} / \mathrm{l}$ vs. $0.849 \pm 0.108 \mu \mathrm{mol} / 1, \mathrm{P}<0.0001)$ in peripheral blood of PD patients were significantly elevated. The expression levels of NLRP3, caspase-1 and IL-1 $\beta$ in mouse astrocytes all increased with the increase of the concentration of oligomerized $\alpha$-synuclein, and Atg5 protein expression also increased gradually with the concentration, and reached the highest level
\end{abstract}

Correspondence to: Dr Xiaohong Wang, The 5th Department of Neurology, the Third Affiliated Hospital of Qiqihar Medical University, 27 Taishun Street, Tiefeng, Qiqihar, Heilongjiang 161000, P.R. China

E-mail: wangxiaohong904@126.com

Key words: $\alpha$-synuclein, autophagy, NLRP3 inflammasome, Parkinson's disease when the concentration was $10 \mu \mathrm{g} / \mathrm{ml}$. The expression levels of NLRP3, caspase-1 and IL-1 $\beta$ were inhibited after the addition of autophagy inhibitor 3-MA. $\alpha$-synuclein mediates the activation of NLRP3 inflammasome in PD patients by upregulating Atg5 protein expression.

\section{Introduction}

Parkinson's disease (PD) is a degenerative neurological disease affected by a variety of comprehensive factors. Its main manifestations include motor symptoms represented as sluggish movement, tremor and paralysis, postural gait disorder and other non-motor symptoms such as depression, which can seriously threaten the quality of life of middle-aged and elderly patients (1). Pathologically, PD is characterized by a severe loss of dopaminergic neurons in the striatum nistriatum, a change that is caused by the accumulation and spread of characteristic Lewy bodies and nerve sheath inclusions due to misfolded fibers (2). These misfolded fibers, mainly derived from a neurotoxic form of $\alpha$-synuclein, can accumulate spontaneously at the junction of brain regions and cause degeneration of dopaminergic neurons $(3,4)$. In recent years, a large number of studies have shown that $\alpha$-synuclein plays an important regulatory role in non-specific immunity of the body. Among them, A53T mutant $\alpha$-synuclein can significantly activate the key transcription factor of inflammatory response, $\mathrm{NF}-\kappa \mathrm{B}$, thereby activating microglia and astrocytes (5). Apart from that, $\alpha$-synuclein can also promote the expression of toll-like receptors (TLRs), such as TLR2, TLR3 and TLR7 (6). As an important component of non-specific immunity, TLR is regularly localized on the surface of cells or internal vesicles, recognizing molecules with conserved structures and stimulating the body to produce an immune response that causes an inflammatory response. Therefore, we highly hypothesized that mutations and abnormal aggregation of $\alpha$-synuclein might have a certain regulatory effect on the inflammatory state of the body.

At present, emerging evidence exhibits that systemic low-level inflammation can accelerate the development of degenerative changes in the brain with increasing age (7), and most men and women aged over 65 years have increased 
serum IL-6, TNF and IL-18 levels $(8,9)$. NLRP3 inflammasome, as a polymeric complex, can bind to caspase-1 and affect the secretion of the inflammatory factor IL-1 $\beta$. Many experiments have proved that its expression is closely related to the onset of age-related diseases, however, its role in the pathogenesis of PD remains poorly understood. While autophagy, acts as an intracellular, homeostatic mechanism, mediates the inactivation or selective clearance of harmful substrates or secondary material signals, such as damaged mitochondria. There is increasing evidence that autophagy exerts vital effect on the innate immune response. In addition, autophagy-related pathways are implicated in the regulation of NLRP3 inflammasome activation (10-12). It has been reported in the literature that $\alpha$-synuclein can promote immune activation in PD patients. Therefore, this study aimed to investigate the mechanism of $\alpha$-synuclein in affecting the progression of PD through autophagy-related pathways.

\section{Materials and methods}

Study subjects. This study was approved by the Medical Ethics Committee of the Third Affiliated Hospital of Qiqihar Medical University (Qiqihar, China), and the experiment was conducted in accordance with the Guide for the Care and Use of Experimental Animals. Peripheral blood samples of 40 PD patients admitted to the department of neurology of the hospital from March 2018 to January 2019 were collected. Inclusion criteria: Patients with the characteristic progressive progression of the disease and 2 or more typical symptoms (including static tremor, sluggish movement, rigidity, postural instability) were included, while those with other neurological tumors were excluded. Further 40 healthy normal people who participated in physical examination were enrolled in the control group. They were matched with PD patients according to age, sex and education level, and those with traumatic brain injury, suspected degenerative disease with Parkinson's disease, Parkinsonrelated diseases (such as Lewy body dementia and Alzheimer's disease), or severe organic or systemic diseases were excluded. All the study subjects were informed of the purpose and the protocol of the study before the blood was drawn, and written informed consent was obtained.

Enzyme-linked immunosorbent assay (ELISA) for the detection of plasma oligomerized, phosphorylated $\alpha$-synuclein and IL-1 $\beta$. Detection method of oligomerized $\alpha$-synuclein: The detection method was carried out in accordance with the established detection method (13). An amount of $100 \mu 1$ of 3D5 monoclonal antibody (Santa Cruz Biotechnology, Inc., sc-47696) with a final concentration of $1 \mu \mathrm{g} / \mathrm{ml}$ was added to each well of the 96-well plate, incubated at $37^{\circ} \mathrm{C}$ for $2 \mathrm{~h}$ for coating, and washed with PBST after culturing overnight at $4^{\circ} \mathrm{C}$. Then each well was incubated at $37^{\circ} \mathrm{C}$ for $2 \mathrm{~h}$ with $200 \mu \mathrm{l}$ of $10 \%$ BSA sealant and then washed with PBST. Then recombinant human $\alpha$-synuclein (rPeptide, s-1005-2) diluted by multiple ratio (concentrations of $0.5,0.25,0.125,0.0625,0.03125$, and $0 \mathrm{~mol} / 1$, respectively) was added with the sample to be tested into each well, incubated at $37^{\circ} \mathrm{C}$ for $2 \mathrm{~h}$ before rinsing with PBST. Then, $100 \mu \mathrm{l}$ of $1 \mathrm{mg} / \mathrm{l}$ avidin (Solarbio, A8280) was added, and the cells were incubated for $2 \mathrm{~h}$ at $37^{\circ} \mathrm{C}$ and washed with PBST. Diluted human avatin-labeled alkaline phosphatase (Solarbio, k0068r-ap) was added, incubated at $37^{\circ} \mathrm{C}$ for $1 \mathrm{~h}$ and then washed with PBST. Finally, $100 \mu 1 \mathrm{pNPP}$ was added to each well, and the color was developed at $37^{\circ} \mathrm{C}$ for $30 \mathrm{~min}$, and the absorbance was measured using a $405 \mathrm{~nm}$ wavelength microplate reader (Thermo Fisher Scientific, Inc., Multiskan FC). Three replicate wells were set for each sample and the experiment was performed three times.

Detection method of phosphorylated $\alpha$-synuclein: $\alpha$-synuclein (p-Ser129) (Abcam, ab51253) monoclonal antibody $(100 \mu \mathrm{l})$ with a final concentration of $0.1 \mathrm{mg} / \mathrm{l}$ was added to each well of the 96-well plate, and the remaining steps were the same as stated above.

Detection method of IL-1 $\beta$, IL- 6 and TGF- $\alpha$ : The collected peripheral blood of the patient was allowed to stand at room temperature for $40 \mathrm{~min}$, and the serum was precipitated and centrifuged at $1,000 \times \mathrm{g}$ for $10 \mathrm{~min}$ at $4^{\circ} \mathrm{C}$. After centrifugation, the light yellow transparent serum from the upper layer was collected and stored frozen at $-80^{\circ} \mathrm{C}$ in a refrigerator for later use. Then the expression levels were measured using IL-1 $\beta$ (ProteinTech Group, Inc., KE00021), IL-6 (ProteinTech Group, Inc., KE00007) and TGF- $\alpha$ (Qiaoyu Biotechnology, QY-MB10203) ELISA kits, and the procedures were in accordance with the kit instructions.

Primary cell isolation and culture. Neonatal 3-4d C57BL/6 mice were sacrificed by spinal dislocation, and the heads of the mice were placed on ice to isolate the cerebral cortex. The primary cell separation method was established as previously described (13). After the cerebral cortex was removed, $0.25 \%$ trypsin (Beyotime, C0201) was used for digestion and cell suspension. Then, 90\% Dulbecco's modified Eagle's medium: Nutrient Mixture F-12 (DMEM/F-12) (HyClone, sh30023.01b) $+10 \%$ fetal bovine serum (FBS) (HyClone, Sv30087.01) $+100 \mu / \mathrm{ml}$ penicillin $+100 \mu / \mathrm{ml}$ streptomycin (Beyotime, C0222) was cultured in a polylysine-coated culture flask for $16 \mathrm{~h}$. According to the different adhesion ability between glial cells, the culture flask was put into a thermostatic oscillator, oscillated at $37^{\circ} \mathrm{C}, 180 \mathrm{rpm}$, for $5 \mathrm{~h}$, and the bottom layer cells were collected as astrocytes with higher purity. Finally, the cells were digested with $0.25 \%$ trypsin, subcultured in a plurality of flasks at 1:2, and cultured to a density of $90 \%$.

Drug treatment of cells. The number of astrocytes was adjusted to $1 \times 10^{7} / \mathrm{ml}$, inoculated in the 6 -well plate, with $3 \mathrm{ml}$ per well. The astrocytes were divided into control group and drug treatment group when they grew to $80 \%$ density. The drug treatment group was divided into low, medium and high concentration groups according to different concentrations of the added drugs, which were 5, 10 and $20 \mu \mathrm{g} / \mathrm{ml}$ of oligomerized $\alpha$-synuclein. Judged by whether autophagy inhibitors were added or not, the cells were divided into a drug treatment group and an autophagy inhibition group. The drug treatment group was treated with $10 \mu \mathrm{g} / \mathrm{ml}$ oligomerized $\alpha$-synuclein, while the autophagy inhibition group was added with $10 \mu \mathrm{g} / \mathrm{ml}$ oligomerized $\alpha$-synuclein and 5 mM 3-MA (MCE, hy-19312), and the culture was continued for $24 \mathrm{~h}$.

Western blot for protein detection. RIPA lysate (Beyotime, P0013C) was applied for cell lysis and centrifugation at $15,000 \mathrm{x} g$ for $15 \mathrm{~min}$ at $4^{\circ} \mathrm{C}$ to extract cell protein. The protein 
A

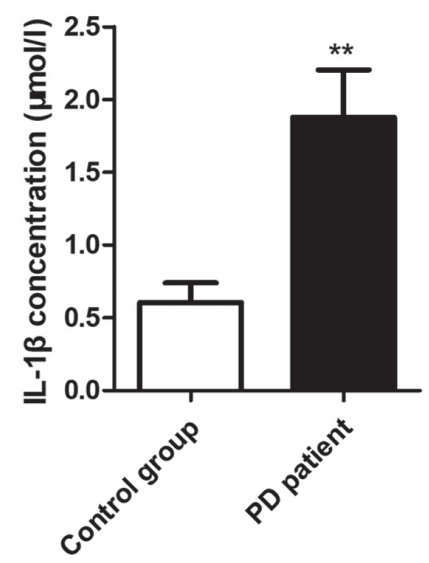

B

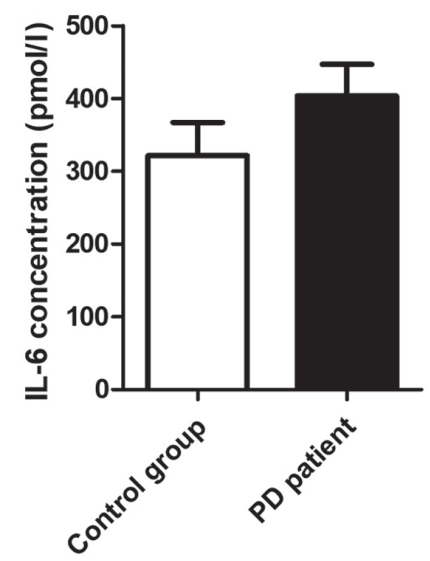

C

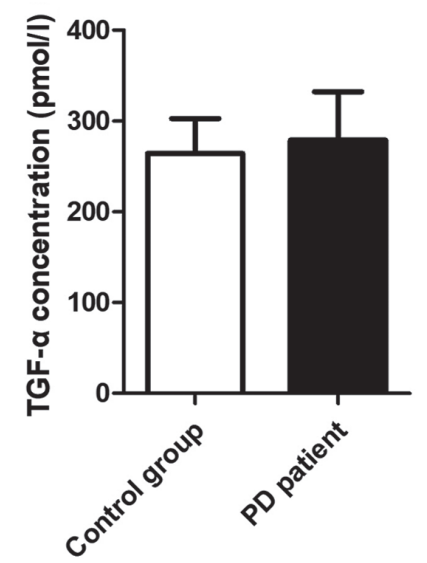

Figure 1. Plasma levels of inflammatory factors IL-1 $\beta$, IL-6 and TGF- $\alpha$ in PD patients. (A) IL-1 $\beta$ expression level in peripheral blood; (B) IL-6 expression level in peripheral blood; (C) TGF- $\alpha$ expression level in peripheral blood. ${ }^{* *} \mathrm{P}<0.01$. IL-1 $\beta$, interleukin- $1 \beta$; IL-6, interleukin-6; TGF- $\alpha$, transforming growth factor- $\alpha$; PD, Parkinson's disease.

A

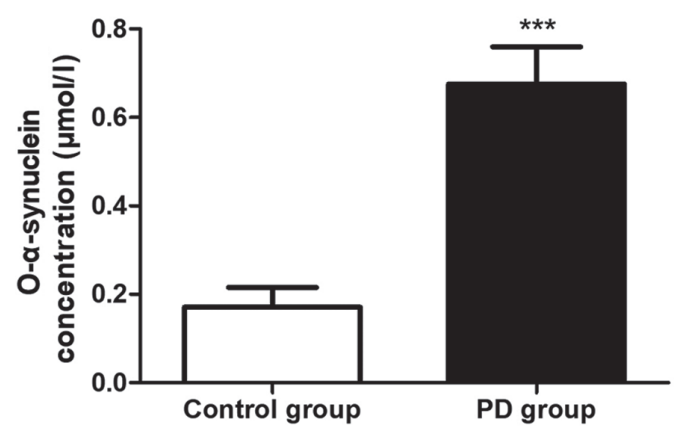

B

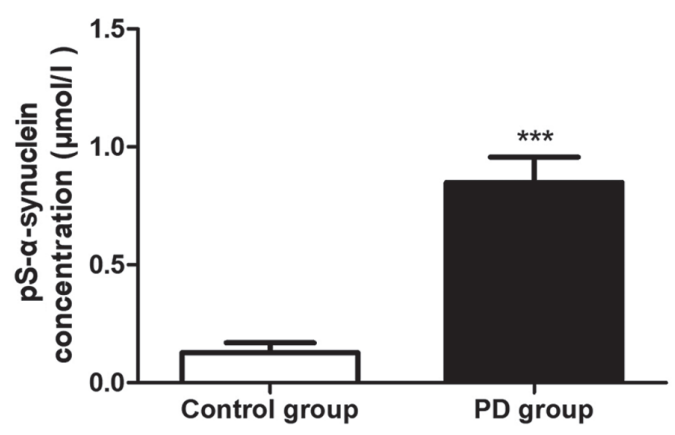

Figure 2. Plasma oligomerized and phosphorylated $\alpha$-synuclein expression levels in PD patients. (A) Expression level of oligomerized $\alpha$-synuclein in peripheral blood; (B) Expression level of phosphorylated $\alpha$-synuclein in peripheral blood. ${ }^{* * *} \mathrm{P}<0.001$. O- $\alpha$-synuclein, oligomerized $\alpha$-synuclein; Ps- $\alpha$-synuclein, phosphorylated $\alpha$-synuclein; PD, Parkinson's disease.

concentration was detected by BCA assay (Beyotime, P0012S) and gel electrophoresis was performed in $15 \%$ SDS-PAGE. Then, the protein was transferred to PVDF membrane by semidry transfer method, and 5\% skim milk powder was adopted to seal the membrane for $1 \mathrm{~h}$. Then, NLRP3 (ProteinTech Group, Inc., 19771-1-AP, 1:1000), caspase-1 (ProteinTech Group, Inc., 22915-1-AP, 1:500), IL-1 $\beta$ (ProteinTech Group, Inc., 16806-1AP, 1:1000) and Atg5 (ProteinTech Group, Inc., 10181-2-AP, 1:1000) were added, respectively, with GAPDH (ProteinTech Group, Inc., 60004-1-Ig, 1:5000) as the internal reference, triple rinsed with $0.1 \%$ PBST after incubation overnight at $4^{\circ} \mathrm{C}$, adding corresponding HRP-labeled goat anti-rabbit (or mouse) immunological secondary antibody (ProteinTech Group, Inc., SA00001-1/2, 1:3000), incubated for $1 \mathrm{~h}$ at room temperature, and washed 3 times with $0.1 \%$ PBST. Finally, ECL chemiluminescence reagent (Beyotime, P0018FS) was employed to develop, fix and photograph (Odyeesy, LI-COR) in the dark, and then it was analyzed using the Corning Axygen gel imaging system.

Statistical analysis. All the collected data were statistically analyzed by SPSS 23.0. The measurement data were expressed as mean \pm standard deviation. Independent t-test was applied for comparison between the two groups, and one-way ANOVA was used for comparison among multiple groups. $\mathrm{P}<0.05$ was considered to indicate a statistically significant difference.

\section{Results}

Elevated plasma inflammation-related factor $I L-1 \beta$ in PD patients. The levels of inflammatory factors in the plasma of PD group and control group were detected by ELISA. It was found that IL-1 $\beta$ was significantly increased in the patient group $(0.604 \pm 0.136 \mu \mathrm{mol} / 1$ vs. $1.876 \pm 0.327 \mu \mathrm{mol} / 1, \mathrm{P}=0.002)$ (Fig. 1A), while the expression levels of IL-6 (321.4 $\pm 45.7 \mathrm{pmol} / 1$ vs. $403.8 \pm 43.19 \mathrm{pmol} / \mathrm{l}, \mathrm{P}=0.206$ ) (Fig. $1 \mathrm{~B})$ and $\mathrm{TGF}-\alpha$ $(264.3 \pm 38.2 \mathrm{pmol} / 1$ vs. $278.8 \pm 53.3 \mathrm{pmol} / \mathrm{l}, \mathrm{P}=0.827)($ Fig. $1 \mathrm{C})$ did not identify any significant increase.

Increased plasma $\alpha$-synuclein expression in PD patients. Plasma $\alpha$-synuclein levels in the PD group and the control group were detected by ELISA, and it was observed that both the expression levels of oligomerized $\alpha$-synuclein $(0.171 \pm 0.044 \mu \mathrm{mol} / 1$ vs. $0.676 \pm 0.083 \mu \mathrm{mol} / 1$, $\mathrm{P}<0.0001$ ) (Fig. 2A) and phosphorylated $\alpha$-synuclein 
A

0

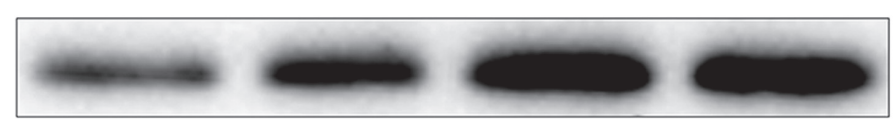

caspase-1

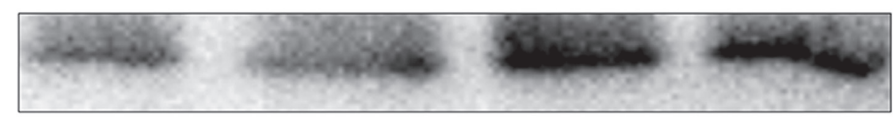

IL-1 $\beta$

GAPDH

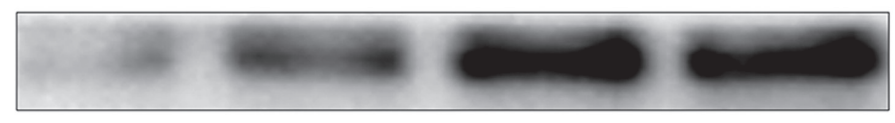

B

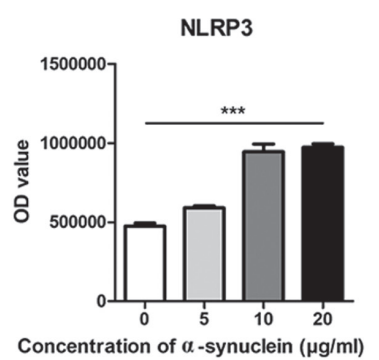

C

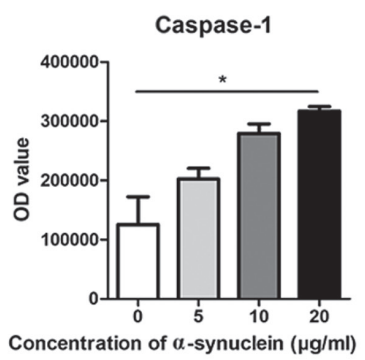

D

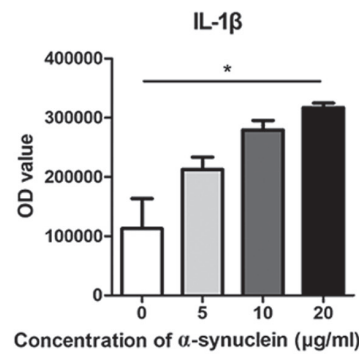

Figure 3. NLRP3 and related molecules in astrocytes at different drug concentrations. (A) Western blot of expression and quantification of (B) NLRP3, (C) caspase-1 and (D) IL-1 $\beta$ protein in mouse astrocytes after adding $0,5,10$ and $20 \mu \mathrm{g} / \mathrm{ml}$ oligomerized $\alpha$-synuclein. IL-1 $\beta$, interleukin-1 $\beta$; NLRP3, Pyrin Domain Containing Protein $3 .{ }^{*} \mathrm{P}<0.05 ;{ }^{* * *} \mathrm{P}<0.001$.

$(0.128 \pm 0.041 \mu \mathrm{mol} / 1$ vs. $0.849 \pm 0.108 \mu \mathrm{mol} / 1, \mathrm{P}<0.0001)$ (Fig. 2B) in the PD group were markedly increased, with statistically significant differences.

Oligomerized $\alpha$-synuclein induction of NLRP3 inflammasome and expression of related molecules in astrocytes. Oligomerized $\alpha$-synuclein with different concentrations of 0 , 5,10 , and $20 \mu \mathrm{g} / \mathrm{ml}$ were added to the astrocytes of primary mice, respectively, and the expression level of NLRP3 was detected by western blot. It was found that NLRP3 expression was elevated with the increase of drug concentration in the cells (Fig. 3A and B). Further detection of other inflammasome related molecular proteins revealed a similar trend in caspase-1 and IL-1 $\beta$ (Fig. 3C and D).

Oligomerized $\alpha$-synuclein affects NLRP3 inflammation by affecting autophagy. After the addition of 0, 5, 10, and $20 \mu \mathrm{g} /$ $\mathrm{ml}$ oligomerized $\alpha$-synuclein, the autophagy-related molecule Atg5 was upregulated in astrocytes with increasing drug concentration (Fig. 4A and B). To further explore whether oligomerized $\alpha$-synuclein causes NLRP3-related inflammatory responses by affecting autophagy, we added oligomerized $\alpha$-synuclein/oligomerized $\alpha$-synuclein + autophagy inhibitors (3-MA) to cells. Western blot results showed that the expression levels of NLRP3, caspase-1 and IL-1 $\beta$ expression were inhibited by 3-MA (Fig. 4C and D), indicating that the activation of NLRP3 inflammatory pathway by oligomerized $\alpha$-synuclein was mediated by autophagy.

\section{Discussion}

As a neurodegenerative disease, Parkinson's disease has been extensively confirmed to be significantly associated with age, but its specific pathogenesis remains a subject of investigation. In recent years, studies have found a positive correlation between age and inflammatory status (7), as serum levels of IL-6, TNF, and IL-18 are elevated in older people over the age of $65(8,9)$. A growing number of studies have found that with the increase of age, a systemic mild inflammatory state can lead to the progression of degenerative changes in the brain, thus forming the 'hypothalamic microinflammation' theory, which mainly believes that under the condition of aging and metabolic syndrome, the abnormal stimulation of the hypothalamus activates the inflammatory signal pathway, leading to the formation of chronic and stable inflammatory background $(14,15)$. The NLRP3 inflammasome acts as a multimeric complex in the cytoplasm and serves as a platform for caspase- 1 activation and promotion of IL-1 $\beta$ maturation (16). It has been found that overexpression is associated with many age-related inflammatory disorders (10). The role of NLRP3 inflammasome in degenerative diseases may be related to their responses to various danger-associated molecule patterns (DAMP), including $\alpha$-synuclein, extracellular adenosine triphosphate (ATP), excessive glucose, ceramide, amyloid, urate and cholesterol crystals (17). In this study, the plasma levels of oligomerized and phosphorylated $\alpha$-synuclein in peripheral blood of patients with PD were both higher than 
A

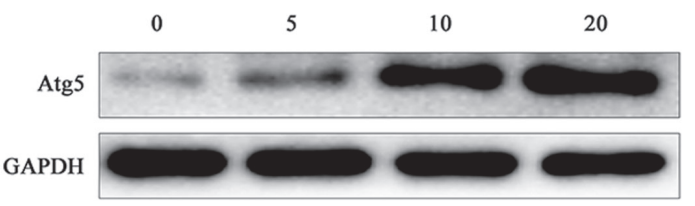

B

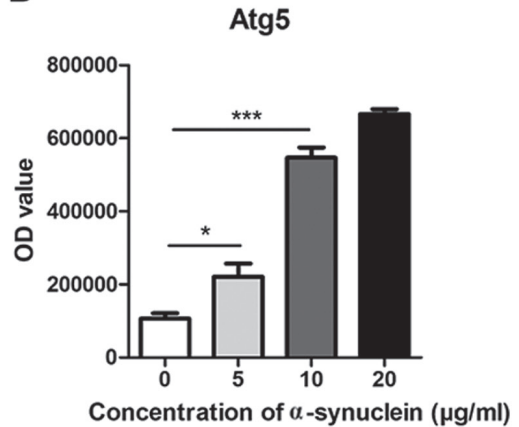

C

$\begin{array}{rrrrr}\alpha \text {-synuclein } & - & - & + & + \\ \text { 3-MA } & - & + & - & +\end{array}$

NLRP3

caspase-1

IL-1 $\beta$
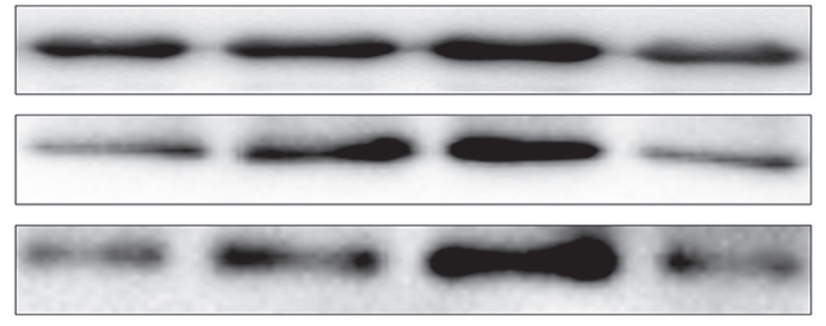

GAPDH

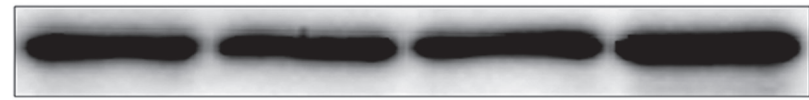

D
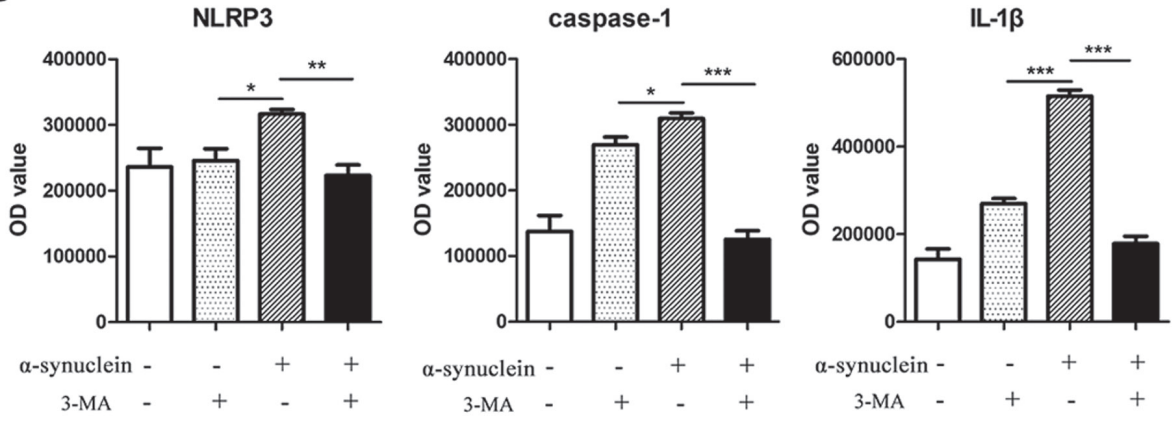

Figure 4. Expression levels of Atg5 and NLRP3 related proteins in mouse astrocytes at different drug concentrations. (A) Western blot of expression of Atg5 protein in mouse astrocytes after adding $0,5,10$, and $20 \mu \mathrm{g} / \mathrm{ml}$ oligomerized $\alpha$-synuclein. (B) Expression and statistical representation of NLRP3, caspase-1 and IL-1 $\beta$ in cells after administration of $10 \mu \mathrm{g} / \mathrm{ml}$ oligomerized $\alpha$-synuclein and/or 3-MA in mouse astrocytes. (C and D) The western blot of expression levels and quantification of NLRP3, caspase-1 and IL-1 $\beta$ expression were inhibited by 3-MA. NLRP3, Pyrin Domain Containing Protein 3, 3-MA, 3-methyladenine; IL-1 $\beta$, interleukin- $1 \beta .{ }^{*} \mathrm{P}<0.05 ;{ }^{* *} \mathrm{P}<0.01 ;{ }^{* * *} \mathrm{P}<0.001$.

those in normal subjects. ELISA method applied to analyze the expression level of inflammatory factors in plasma of PD patients, revealed that the expression level of IL-1 $\beta$ was significantly increased, while there was no significant difference in IL- 6 and TGF- $\alpha$. These result suggests that $\alpha$-synuclein in PD patients may activate NLRP3 inflammatory factors to release excess IL-1 $\beta$, thus leaving the patient in a state of chronic mild inflammation.

The characteristic pathological changes of PD are Lewy body formation and dopaminergic neuropathy, of which the latter often occurs in the late stage of the disease, when the neurodegeneration rate can be as much as $70 \%$ (18). The formation of Lewy body is usually much earlier than that of dopaminergic neuropathy, so the early Lewy body formation is the best target for disease occurrence and intervention. The main component of Lewy body is abnormally aggregated $\alpha$-synuclein. Studies have shown that $\alpha$-synuclein can be released from neurons into extracellular fluids, including cerebrospinal fluid and plasma. In the current study, ELISA was used to find a significant increase in plasma $\alpha$-synuclein expression in PD patients. $\alpha$-synuclein normally exists in presynaptic nerve terminals and mediates normal physiological functions (19). In special cases, its neurotoxic abnormal metabolites are released into the extracellular matrix, which can activate glial cells and innate immune responses, leading to neuronal damage (20-22). While in turn, neurons can be 
internalized by neurotoxic $\alpha$-synuclein through endocytosis to avoid extensive damage, but long-term pathological accumulation of $\alpha$-synuclein will result in apoptosis or degeneration of neuronal cells (21). A study demonstrated that the overexpression of $\alpha$-synuclein in rodent models (23) without obvious toxicity could impair the release of the neurotransmitters glutamate and dopamine. Through direct imaging of the synaptic vesicle circulation process, it was found that the synaptic vesicle circulation pool was significantly reduced, and ultrastructural analysis exhibited that the density of synaptic vesicles in the active area was decreased. Imaging further revealed the defect of reaggregation of synaptic vesicles after endocytosis. Therefore, the increased level of $\alpha$-synuclein in PD patients will lead to a special physiological defect in the process of synaptic vesicle regeneration, which precedes the detectable neuropathology and provides a solid theoretical basis for further exploration of the influence of $\alpha$-synuclein on the progress of PD disease. In the present study, mouse astrocytes were extracted in the primary generation, and different concentrations of oligomerized $\alpha$-synuclein were added to the culture medium. The activation of NLRP3-related signaling pathway was detected by western blot, and significant activation of NLRP3, caspase-1 and IL-1 $\beta$ was observed. Combined with a large number of literature and the results of this study, it can be concluded that $\alpha$-synuclein plays an important regulatory role in non-specific immune responses and is of great significance in the pathogenesis of neurodegenerative diseases.

Some studies have analyzed the autopsy of brain tissue of PD patients, and the results showed that there was obvious microglial cell activation and glial cell invasion in the regions with degeneration of dopaminergic neurons (24). The reason is that abnormal lipids, proteins and metabolic stress factors accumulated in tissues and neurons of PD patients (such as $\alpha$-synuclein or mitochondrial dysfunction) could send stress signals to small glial cells and astrocytes in the environment (23). Others (25) have reported that when severe metabolic and lipid disorders occur in neurons, fatty acids and other molecules can directly participate in the activation of neuronal inflammation in brain tissue. In recent years, researchers have found that $\alpha$-synuclein activates microglia activation and produces neuroinflammation in neurodegenerative diseases such as PD and other related diseases. A study (26) displayed that the combined application of wild-type $\alpha$-synuclein and toll-like receptor stimulation (TLR) in the microglia of primary mice could significantly increase the secretion levels of IL-6, McP-1 and CXCL10. In addition, it showed that microglia stimulated by $\alpha$-synuclein could simultaneously differentiate into M1/M2 intermediate phenotype. According to some other studies, $\alpha$-synuclein upregulates the expression of immunosome-regulating receptors TLR2, TLR3, and TLR7 $(5,6)$, and activates NF- $\mathrm{B}$, a key transcription factor for inflammation. Previous studies mainly focused in microglia and neuronal cells. In this study, astrocytes from mouse brain tissue were extracted for testing, and we found that $\alpha$-synuclein could activate NLRP3 inflammasome through autophagy signaling pathway and promote the occurrence of chronic inflammatory state, which was also consistent with the previous results. Moreover, a study on traumatic brain injury also revealed that trauma could cause severe brain inflammation and significantly increase the level of $\alpha$-synuclein in the brain (27), increasing the risk of PD, further validating the relationship between inflammation and Parkinson's disease. Most of the studies were designed to investigate the promotion of normal or abnormal structural $\alpha$-synuclein levels by different stimuli. However, there is still scarce research on the influence of abnormally high expression of oligomerized $\alpha$-synuclein on brain tissue. In this study, combined with previous literature reports, we found that there was a mutual regulatory relationship between inflammation and $\alpha$-synuclein.

The relationship between inflammation and PD has long been elucidated. The 1918 influenza pandemic caused encephalitis, and some patients further developed PD, which was then called Von Ikonomo disease (28). Since then, a significant link between inflammation and Parkinson's disease has been found by studying other viral types, in which mice with H5N1 infection showed that the virus caused microglia activation from peripheral infection to the central nervous system, and $\alpha$-synuclein polymerization and death of dopaminergic neurons in the substantia nigra. Whereas, previous studies believed that inflammatory response and activation of immune cells are secondary processes in PD. In recent years, studies have clarified that inflammation can directly participate in the pathogenesis of PD. First, the expression levels of pro-inflammatory factors, including TNF- $\alpha$, IL-1 $\beta$, IL-6 and TLR, were increased in PD brain tissue. Second, injection of PD-associated toxins (MPTP and 6-hydroxydopamine) in a mouse experimental model could cause significant inflammation in the brain tissue after injection and lead to degeneration of dopaminergic neurons $(29,30)$. However, intracerebral injection of lipopolysaccharide (LPS) can induce intracellular accumulation of insoluble polymeric $\alpha$-synuclein (31), reducing the resistance of dopamine neurons to MPTP and 6-hydroxydopamine (32), which is mainly related to the activation of related proinflammatory factors, especially IL-1 $\beta$ (33). Accorded with previous results, the addition of IL-1 $\beta$ receptor antagonists significantly reduced TNF- $\alpha$ and IFN- $\gamma$ expression levels and abolished LPS-mediated dopamine neuronal death below toxic doses (34). In another model of inflammation, injection of the TLR3 agonator polyglucoside:polycysteine [poly(I:C)] into the substantia nigra of mice led to persistent inflammation, increased sensitivity to low doses of 6-hydroxydopamine, and interfered with proteins involved in synaptic transmission and axonal transport (35). Compared with the normal control group, the expression of TLR2 and TLR9 in the brain of PD patients was increased, and the expression of TLR2 was positively correlated with the accumulation of $\alpha$-synuclein (36) in the body.

Furthermore, it has been reported that $\alpha$-synuclein stimulation can significantly activate inflammatory monocytes and microglia in mice, and promote PD disease progression by producing excessive inflammatory responses $(5,6)$. Moreover, studies have indicated that autophagy is closely related to immune cells and may be involved in the pathogenesis of many diseases, including neurodegeneration, metabolic disorders and aging-related diseases. For example, autophagy is reported to participate in the negative regulation of NLRP3 related signaling pathways, and the product of mitochondrial reactive oxygen species (37) and autophagosome dysfunction, 
cathepsin B (38), can be used as a 'second signal' to stimulate the activation of NLRP3 inflammasome. Previously, it was exhibited that the exposure of methamphetamine (METH) to $\alpha$-synuclein significantly reduced chaperonmediated autophagy activity, and the decreased lamp-2a expression could alleviate neurotoxic reactions induced by $\alpha$-synuclein (39). On the contrary, overexpression of the heat shock protein 70 (HSP70) reduced abnormally aggregated $\alpha$-synuclein and inhibited apoptosis induced by METH exposure. A study on PD (40) suggested that the addition of autophagy small molecule inhibitors in cell experiments and animal models could inhibit the formation of NLRP3PYCARD-CASP1 complex, thereby delaying the progression of PD. In line with previous studies, we found that the expression of autophagy associated molecule Atg5 was significantly increased when stimulated by $\alpha$-synuclein, and autophagy inhibitor 3-MA inhibited $\alpha$-synuclein-mediated activation of NLRP3-related molecules. $\alpha$-synuclein, as an immunogenic substance, is usually an abnormal metabolite of nerve cells, which activates the NLRP3 inflammasome pathway through the autophagy-related molecule Atg5.

We did not yet carry out in vivo experiments in mice, nor did we explore the activation of $\alpha$-synuclein and autophagy on the inflammatory response in vivo, or the dynamic process of the changes of Lewy body and dopaminergic neurons in the substantia nigra. Thus, further studies are anticipated. By examing $\alpha$-synuclein and inflammation-related factors in peripheral blood of PD patients, this study not only verified the activation effect of $\alpha$-synuclein on NLRP3-related molecules and autophagy in cells, but also found that the application of autophagy inhibitor 3-MA could significantly inhibit the inflammatory pathway, providing a solid basis for autophagy inhibitors to be potential targets for PD treatment.

\section{Acknowledgements}

Not applicable.

\section{Funding}

This study was supported by Mandatory Scientific Research Project of Qiqihar Science and Technology Bureau (SFGG201949).

\section{Availability of data and materials}

The datasets used and/or analyzed during the present study are available from the corresponding author on reasonable request.

\section{Authors' contributions}

$\mathrm{XW}, \mathrm{JC}, \mathrm{DH}$ and LD led the conception and design of this study. XW, JC, XZ, LJ, YY and FG were responsible for the data collection and analysis. DH, LD and YY were in charge of interpreting the data and drafting the manuscript. XW and DH made revision from critical perspective for important intellectual content. All authors read and approved the final manuscript.

\section{Ethics approval and consent to participate}

The study was approved by the Ethics Committee of the Third Affiliated Hospital of Qiqihar Medical University (Qiqihar, China). Signed informed consents were obtained from the patients and/or the guardians.

\section{Patient consent for publication}

Not applicable.

\section{Competing interests}

The authors declare that they have no competing interests.

\section{References}

1. Scheperjans F, Aho V, Pereira PA, Koskinen K, Paulin L, Pekkonen E, Haapaniemi E, Kaakkola S, Eerola-Rautio J, Pohja M, et al: Gut microbiota are related to Parkinson's disease and clinical phenotype. Mov Disord 30: 350-358, 2015.

2. Poewe W, Seppi K, Tanner CM, Halliday GM, Brundin P, Volkmann J, Schrag AE and Lang AE: Parkinson disease. Nat Rev Dis Primers 3: 17013, 2017.

3. Brettschneider J, Del Tredici K, Lee VM and Trojanowski JQ: Spreading of pathology in neurodegenerative diseases: A focus on human studies. Nat Rev Neurosci 16: 109-120, 2015.

4. Luk KC, Kehm V, Carroll J, Zhang B, O'Brien P, Trojanowski JQ and Lee VM: Pathological $\alpha$-synuclein transmission initiates Parkinson-like neurodegeneration in nontransgenic mice. Science 338: 949-953, 2012

5. Goldberg EL and Dixit VD: Drivers of age-related inflammation and strategies for healthspan extension. Immunol Rev 265: 63-74, 2015.

6. Pedersen M, Bruunsgaard H, Weis $\mathrm{N}$, Hendel HW, Andreassen BU, Eldrup E, Dela F and Pedersen BK: Circulating levels of TNF-alpha and IL-6-relation to truncal fat mass and muscle mass in healthy elderly individuals and in patients with type-2 diabetes. Mech Ageing Dev 124: 495-502, 2003.

7. Ferrucci L, Corsi A, Lauretani F, Bandinelli S, Bartali B, Taub DD, Guralnik JM and Longo DL: The origins of age-related proinflammatory state. Blood 105: 2294-2299, 2005.

8. Patel MN, Carroll RG, Galván-Peña S, Mills EL, Olden R, Triantafilou M, Wolf AI, Bryant CE, Triantafilou K and Masters SL: Inflammasome priming in sterile inflammatory disease. Trends Mol Med 23: 165-180, 2017.

9. Lu A, Magupalli VG, Ruan J, Yin Q, Atianand MK, Vos MR, Schröder GF, Fitzgerald KA, Wu H and Egelman EH: Unified polymerization mechanism for the assembly of ASC-dependent inflammasomes. Cell 156: 1193-1206, 2014.

10. Guo C, Xie S, Chi Z, Zhang J, Liu Y, Zhang L, Zheng M, Zhang X, Xia D, Ke Y, et al: Bile acids control inflammation and metabolic disorder through inhibition of NLRP3 inflammasome. Immunity 45: 944, 2016.

11. Matsuo K, Cheng A, Yabuki Y, Takahata I, Miyachi H and Fukunaga K: Inhibition of MPTP-induced $\alpha$-synuclein oligomerization by fatty acid-binding protein 3 ligand in MPTP-treated mice. Neuropharmacology 150: 164-174, 2019.

12. Zhang Y, Kim MS, Jia B, Yan J, Zuniga-Hertz JP, Han C and Cai D: Hypothalamic stem cells control ageing speed partly through exosomal miRNAs. Nature 548: 52-57, 2017.

13. Zhang G, Li J, Purkayastha S, Tang Y, Zhang H, Yin Y, Li B, Liu $\mathrm{G}$ and Cai D: Hypothalamic programming of systemic ageing involving IKK- $\beta$, NF- $\kappa$ B and GnRH. Nature 497: 211-216, 2013.

14. Lamkanfi M and Dixit VM: Mechanisms and functions of inflammasomes. Cell 157: 1013-1022, 2014.

15. Youm YH, Grant RW, McCabe LR, Albarado DC, Nguyen KY, Ravussin A, Pistell P, Newman S, Carter R, Laque A, et al: Canonical Nlrp3 inflammasome links systemic low-grade inflammation to functional decline in aging. Cell Metab 18: 519-532, 2013.

16. Gershanik OS: Past, present, and future of Parkinson's disease. Mov Disord 32: 1263, 2017 
17. Norris EH, Giasson BI and Lee VM: Alpha-synuclein: Normal function and role in neurodegenerative diseases. Curr Top Dev Biol 60: 17-54, 2004.

18. Ma QL, Chan P, Yoshii M and Uéda K: Alpha-synuclein aggregation and neurodegenerative diseases. J Alzheimers Dis 5: 139-148, 2003.

19. Lee HJ, Suk JE, Patrick C, Bae EJ, Cho JH, Rho S, Hwang D, Masliah E and Lee SJ: Direct transfer of $\alpha$-synuclein from neuron to astroglia causes inflammatory responses in synucleinopathies. J Biol Chem 285: 9262-9272, 2010.

20. Ma D, Jin S, Li E, Doi Y, Parajuli B, Noda M, Sonobe Y, Mizuno T and Suzumura A: The neurotoxic effect of astrocytes activated with toll-like receptor ligands. J Neuroimmunol 254: 10-18, 2013.

21. Abdelkarim H, Marshall MS, Scesa G, Smith RA, Rue E, Marshall J, Elackattu V, Stoskute M, Issa Y, Santos M, et al: $\alpha$-Synuclein interacts directly but reversibly with psychosine: Implications for $\alpha$-synucleinopathies. Sci Rep 8: 12462, 2018.

22. Mori F, Tanji K, Yoshimoto M, Takahashi H and Wakabayashi K: Demonstration of $\alpha$-synuclein immunoreactivity in neuronal and glial cytoplasm in normal human brain tissue using proteinase $\mathrm{K}$ and formic acid pretreatment. Exp Neurol 176: 98-104, 2002.

23. Deleidi M and Isacson O: Viral and inflammatory triggers of neurodegenerative diseases. Sci Transl Med 4: 121ps3, 2012

24. McGeer PL, Itagaki S, Boyes BE and McGeer EG: Reactive microglia are positive for HLA-DR in the substantia nigra of Parkinson's and Alzheimer's disease brains. Neurology 38: 1285-1291, 1988

25. Park J, Wang Q, Wu Q, Mao-Draayer $\mathrm{Y}$ and $\mathrm{Kim} \mathrm{CH} 4$ Bidirectional regulatory potentials of short-chain fatty acids and their G-protein-coupled receptors in autoimmune neuroinflammation. Sci Rep 9: 8837, 2019

26. Roodveldt C,Labrador-Garrido A, Gonzalez-Rey E, Lachaud CC, Guilliams T, Fernandez-Montesinos R, Benitez-Rondan A Robledo G, Hmadcha A, Delgado M, et al: Preconditioning of microglia by $\alpha$-synuclein strongly affects the response induced by toll-like receptor (TLR) stimulation. PLoS One 8: e79160, 2013.

27. Uryu K, Chen XH, Martinez D, Browne KD, Johnson VE, Graham DI, Lee VM, Trojanowski JQ and Smith DH: Multiple proteins implicated in neurodegenerative diseases accumulate in axons after brain trauma in humans. Exp Neurol 208: 185-192, 2007.

28. Picard F, de Saint-Martin A,SalmonE,HirschEand Marescaux C: Postencephalitic stereotyped involuntary movements responsive to L-Dopa. Mov Disord 11: 567-570, 1996.

29. Ros-Bernal F, Hunot S, Herrero MT, Parnadeau S, Corvol JC, Lu L, Alvarez-Fischer D, Carrillo-de Sauvage MA, Saurini F, Coussieu C, et al: Microglial glucocorticoid receptors play a pivotal role in regulating dopaminergic neurodegeneration in parkinsonism. Proc Natl Acad Sci USA 108: 6632-6637, 2011.
30. Depino AM, Earl C, Kaczmarczyk E, Ferrari C, Besedovsky H, del Rey A, Pitossi FJ and Oertel WH: Microglial activation with atypical proinflammatory cytokine expression in a rat model of Parkinson's disease. Eur J Neurosci 18: 2731-2742, 2003.

31. Gao HM, Kotzbauer PT, Uryu K, Leight S, Trojanowski JQ and Lee VM: Neuroinflammation and oxidation/nitration of alphasynuclein linked to dopaminergic neurodegeneration. J Neurosci 28: 7687-7698, 2008

32. Fan LW, Tien LT, Lin RC, Simpson KL, Rhodes PG and Cai Z: Neonatal exposure to lipopolysaccharide enhances vulnerability of nigrostriatal dopaminergic neurons to rotenone neurotoxicity in later life. Neurobiol Dis 44: 304-316, 2011.

33. Pott Godoy MC, Tarelli R, Ferrari CC, Sarchi MI and Pitossi FJ: Central and systemic IL-1 exacerbates neurodegeneration and motor symptoms in a model of Parkinson's disease. Brain 131: 1880-1894, 2008.

34. Koprich JB, Reske-Nielsen $\mathrm{C}$, Mithal $\mathrm{P}$ and Isacson $\mathrm{O}$ : Neuroinflammation mediated by IL-1beta increases susceptibility of dopamine neurons to degeneration in an animal model of Parkinson's disease. J Neuroinflammation 5: 8, 2008.

35. Deleidi M, Hallett PJ, Koprich JB, Chung CY and Isacson O: The Toll-like receptor-3 agonist polyinosinic:polycytidylic acid triggers nigrostriatal dopaminergic degeneration. J Neurosci 30 : 16091-16101, 2010.

36. Dzamko N, Gysbers A, Perera G, Bahar A, Shankar A, Gao J, $\mathrm{Fu} \mathrm{Y}$ and Halliday GM: Toll-like receptor 2 is increased in neurons in Parkinson's disease brain and may contribute to alpha-synuclein pathology. Acta Neuropathol 133: 303-319, 2017.

37. Zhong Z, Umemura A, Sanchez-Lopez E, Liang S, Shalapour S, Wong J, He F, Boassa D, Perkins G, Ali SR, et al: NF- $\kappa$ B restricts inflammasome activation via elimination of damaged mitochondria. Cell 164: 896-910, 2016.

38. Qiao C, Yin N, Gu HY, Zhu JL, Ding JH, Lu M and Hu G: Atp13a2 deficiency aggravates astrocyte-mediated neuroinflammation via NLRP3 inflammasome activation. CNS Neurosci Ther 22: 451-460, 2016.

39. Sun L, Lian Y, Ding J, Meng Y, Li C, Chen L and Qiu P: The role of chaperone-mediated autophagy in neurotoxicity induced by alpha-synuclein after methamphetamine exposure. Brain Behav 9: e01352, 2019.

40. Han X, Sun S, Sun Y, Song Q, Zhu J, Song N, Chen M, Sun T, Xia M, Ding J, et al: Small molecule-driven NLRP3 inflammation inhibition via interplay between ubiquitination and autophagy: Implications for Parkinson disease. Autophagy 15: 1860-1881, 2019 